\title{
Airborne environmental health hazards in children
}

\author{
Pradeep Kumar Gupta
}

Professor and Head, Dept. of Pediatrics, Subharti Medical College, Meerut, Uttar Pradesh, India

*Corresponding Author:

Email: drgupta_jsr@rediffmail.com

\begin{abstract}
Air pollution both of indoor air and ambient air is an important source of morbidity and mortality in children Children are particularly vulnerable due to longer life span so that late manifestations appear clinically as well as behavioural aspects like increased hand-mouth activity. Common indor pollutants are tobacco smoke, mercury moulds and allergens while ambient air pollution is mainly due to particulate matter, lead from automobiles and oxides of suphur and nitrogen.
\end{abstract}

Keywords: Air pollution- ambient and indoor air pollution, Children, Tobacco smoke, mercury, Allergens, Lead, Particulate air particles

Humans need food, water and air for their survival and wholesomeness of these items is important for a good quality of life. According to World Health Organization (WHO) globally, 7 million deaths were attributable to the joint effects of household (HAP) and ambient airpollution (AAP) in 2016. The South East Asian region had most of the burden with 2.4 milliondeaths. ${ }^{1}$ There is extensive literature on health aspects elevated levels of particulate air pollutants of $<10 \mathrm{u}$ and $<2.5 \mathrm{um}$ size and Indian cities have mostly been among the most heavily polluted. ${ }^{2}$ Other inhaled health hazards include ozone, Sulphur-dioxide, nitrogen-dioxide, carbon mono-oxide as well as infectious agents. ${ }^{3}$

Some airborne toxic items are natural products which become hazardous if they exceed a certain level while others have been added as man made products to the environment. Items like mercury vapour, lead, tobacco smoke have been linked to adverse health items like respiratory, cardiac and neurodevelopmental disorders; endocrine disruptions and respiratory inefficiency. Some of these are potential carcinogens as well $^{4}$

\section{Vulnerability of Children}

Persons of all ages are affected by air pollution, but, children are particularly vulnerable due to various reasons. Factors for increased exposure include. ${ }^{5}$

1. Their low body weight, as result they have greater risk for same amount of toxin

2. Greater likely hood of contact as they are closer to the ground where many toxins are concentrated

3. Their higher respiratory rates which create greater opportunity for toxins and infectious agents to enter body and

4. Increased hand-mouth behaviour which predisposes them to ingestion of toxins in dust.

In addition to greater risk of exposure, children are also at higher risk of adverse effects of various toxins due factors like (a) less mature metabolic processes to detoxify the toxins (b) longer future which gives toxins longer time to cause damage from exposure suffered in pregnancy, infancy or childhood and (c) children are in the process of growth and development and these processes create a window of vulnerability

Effects of toxins may be manifesting in various ways such as anthropometric, cognitive or neurodevelopmental in early years and reproductive or immune systems in later childhood and adolescence which may lead to chronic diseases and reproductive inefficiency.

\section{Indoor Air Quality}

Children spend most of their time inside home or school and therefore quality of air they breathe at these places has a profound effect. Several indoor pollutants are known but maximum attention has been given to carbon monoxide, tobacco smoke, biologic contaminants like molds and mildew, lead and pesticides. ${ }^{6}$

Environmental Tobacco Smoke (ETS): Most well known of the all, environmental tobacco smoke is an important health hazard and has relationship with as thma, sudden infant death syndrome (SIDS) and other respiratory tract problems including lung carcinoma in alter life. ${ }^{7}$

Tobacco smoke exposure may occur in home, motor vehicle, school, relatives' homes and care giving facilities. It remains a big risk for asthma, -acute episodes, nocturnal as thma, increased emergency visits to hospital, worsened severity and compromised lung function. ${ }^{8}$

Many studies have identified higher probability of SIDS in association with tobacco use by mother during pregnancy. ${ }^{9}$ Other diseases influenced by ETS include higher risk of middle ear effusions, lower respiratory tract infections like pneumonia and bronchiolitis, persistent runny nose and chronic cough. ${ }^{8}$

The only effort that helps in exposure of ETS to children is prevention. Best is primary prevention by social campaigns to limit initiation. Other important 
intervention is to prevent passive smoking by asking adults not to smoke inside house or in transport vehicle. Carbon Monoxide (CO): Carbon dioxide is a truly silent killer and frequently not suspected. Sources include wood and coal burning, charcoal grills, gas ovens and automobiles, later being an important cause in a situation of incomplete combustion due to poor vehicle maintenance and inadequate ventilation. Poor ventilation is an important contributor in majority of instances. Symptoms are vague like headache, dizziness, nausea, confusion and disorientation. Impaired oxygen transport results in tissue hypoxia causing cerebral symptoms and often lethal arrythmia. Even levels of carboxyhemoglobin as low as 5\% cause symptoms and levels beyond $60 \%$ are possibly fatal. ${ }^{10}$ Prompt suspicion, removing the offending item, oxygen may be of life saving value.

Mercury (Hg) ${ }^{11}$ : Elemental mercury is liquid or vapor at room temperature with a long history of medicinal use as antibacterial, antiseptic, dermatologic ointments, dental amalgams, thermometers, barometers, sphygmomanometers and as preservative (thimerosal) in vaccines etc. Major sources of atmospheric mercury include burning of high Sulphur coal, fluorescent lights, button batteries, medical and industrial waste incineration and fish and other sea food.

Exposure to mercury vapor may occur in home as well as school, may be acute or chronic and as it is uncharged lipid soluble atom, it rapidly distributes in body with half-life of 36-100 days and easily reaches brain and has the ability to cross placenta and reach fetus. Organ damage occurs in brain, kidney and lungs. Children are at greater risk as mercury vapor tends to sit on the floor. Symptoms include metallic taste in mouth, fever, respiratory symptoms of cough and chest tightness, vomiting and diarrhoea. Higher exposure may lead to acute tubular necrosis and acute necrotizing bronchitis.

Chronic exposure to mercury vapor primarily results in neurological effects in CNS due to deposition in cerebral and cerebellar cortices and which may develop months later. Symptoms include insomnia, tremors, delusions, hallucinations, loss of memory, headache, peripheral neuropathy, tubular vision and ataxia. Autonomic dysfunction may cause excessive salivation and sweating.

Effects on fetus and new born from prenatal/ postnatal exposure of the mother using products such as skin lightening cream are unstudied, but fetus may be at risk. Pink disease or acrodynia and erethism have been reported in infants following chronic exposure. An association between thimerosal as vaccine preservative and autism spectrum disorder has been suggested.

In the management of $\mathrm{Hg}$ poisoning, identification and removal of source is very crucial. While organic mercury (fish consumption) is concentrated in the red blood cells and mostly excreted into bile and feces, inorganic mercury is accumulated in kidneys and excreted via urine. Hence, measurement of whole blood levels and renal functions may be useful respectively. In mercury intoxicated symptomatic patients, immediate chelation therapy is indicated. Other indications are not well established. Chelators increase excretion in urine but their effectiveness in reducing toxicity in patient is not certain. Commonly used chelation agents are dimercaprol (BAL) and Dpenicillamine. But BAL is absolutely contraindicated for organic $\mathrm{Hg}$ poisoning due to enhancing of risk of brain levels.

Allergens and Molds ${ }^{12}$ : Molds or fungi often grow in homes with dampness. Fungal components can become airborne either by air currents of mechanical dis ruption. They cause illness by immunologic (most common), toxic and infective mechanisms. Immunological mechanisms include mold specific serum IgE (immediate hypersensitivity), antigen-specific antibody complexes and delayed hypersensitivity. Molds sensitization occurs in genetically predisposed individuals in response to recurrent or chronic environmental exposure. Common fungi causing illness include Candida, Aspergillus, Alternaria and Cladosporium and illnesses due to them include asthma, allergic broncho-pulmonary mycosis, rhinoconjunctivitis, atopic dermatitis. Treatment may include antigen specific immunotherapy, avoidance of allergen, treatment of asthma and oral corticosteroids for refractory lung disease. Allergic fungal sinusitis has also been reported due to Aspergillus which may often be invasive. Treatment usually consists of endoscopic evacuation with debridement, irrigation with antifungal antibiotics in saline and topical use of corticosteroids for long time.

Other important allergens include pets particularly those with fur and dander, dust mite and cockroach.

Particulates and Chemicals: In low income settings, oil, wood and animal dung may be used for heating and cooking which in turn create particulate pollution in the home. This is associated with upper and lower respiratory tract illnesses as also eye diseases.

Volatile organic chemicals are widely used in items like paints and varnishes, cosmetics and furniture made of particle board or pressed board. They all are associated with adverse health outcomes.

Outdoor Air Pollutants: Ambient air or outdoor air pollution has become a big problem in many parts of world including major Indian cities. ${ }^{2}$ Maximum pollution is due to ozone, particulate matter, lead, Sulphur dioxide, $\left(\mathrm{SO}_{2}\right)$, acid aerosols and nitrogen dioxide $\left(\mathrm{NO}_{2}\right)$

Ozone: Ozone is a highly oxidant gas that reacts with respiratory tract membrane. It not directly released but formed by atmospheric reaction from precursor volatile organic compounds and $\mathrm{NO}_{2}$ in presence of sun light. Precursor emissions are mostly derived from diesel consuming transport vehicles and electrical generators. 
Ozone affects macrophage processing of foreign proteins and may enhance sensitization when an atopic child is exposed in presence of common antigens.

Lead $(\mathbf{P b}) .^{\mathbf{1 0 , 1 3}}$ : Definition of childhood lead poisoning has changed over years. As no threshold levels has been identified, there are no safe blood levels for lead but levels of $>10$ microgram per deciliter are viewed with concern as per CDC although they may be neither normal nor safe.

Lead has been widely used since ancient times in medicines, utensils-glass as well as metal, plumbing, paints and pigments, sheets for ships, roofs, gutters, containers, cosmetics, printing types, protective coating, insecticides, batteries, ceramics, lubricants plastics and in modern era in gasoline or petrol. Lead containing pans, utensils or glazed pottery are a big and often overlooked hazard. Till addition of tetraethyl lead to gasoline in December 1921, lead pollution was mainly from indoor use, and thereafter, there has been huge AAP pollution due to release of lead in atmosphere. This lead gets deposited in the soil. Children's risk correlates with their outdoor activity, their hand-to-mouth behaviours and oral exploratory habits and blood levels peak between 18-30 months of age but children with pica are at increased risk for continued lead exposure and so are the children with autism.

Pregnant and lactating women exposed to lead hazard have a high risk for their fetus and infants to develop lead toxicity.

Lead toxicity-plumbism as it is called- causes neurotoxicity from tow mechanisms. They are interference with synaptic neurotransmission and interference with cell adhesion molecules causing disruption to cell migration during critical periods of central nervous system development. Clinical implications include cognitive losses, aggression, hyperactivity and learning failure. It has been estimated that there is a decline of about 10 points in IQ over arrange of serum lead levels ranging from 1-30 $\mu \mathrm{g} / \mathrm{dL}$. Almost all domains-memory, reading, mathematics, fine and gross motor - may be affected.

Lead also interferes with heme synthesis in red blood cells leading to microcytic hypochromic anemia and causes disruption of $\mathrm{T}$ cell function. Lead affects vitamin hydroxylation in kidneys, is toxic to osteoblasts, inhibits new bone vascularization thus promoting osteopenia, osteoporosis and defective hydroxyapatite lattice formation. It also causes poor physical growth and reproductive inefficiency. It has also been implicated in hypertension and renal dysfunction in adults.

Measures to control $\mathrm{Pb}$ poisoning include removing lead from house paints, use of non-lead utensils and low lead gasoline. Chelation with dimercaprol, or Calcium disodium EDTA or Dpenicillamine has been advocated to decrease blood lead concentrations and increase urinary excretion.in children with blood lead levels $45 \mu \mathrm{g} / \mathrm{dL}$ or more. Besides if child has blood levels more than $20 \mu \mathrm{g} / \mathrm{dL}$ neurodevelopmental surveillance should be included in long range management plan.

\section{Particulate Matter}

Particulate matter commonly called as soot is term used to describe a variety of microscopic particles in air. It is characterized by its physical properties. It is mixture of solid particles and liquid droplets in air including dirt, dust and smoke and cigarette fumes and also byproducts of burning. Sources include factories, power plants, motor vehicles -especially diesel trucks, construction activities and wind-blown dust. It is distinguished by particulate size-less than $10 \mu \mathrm{m}$ diameter and less than $2.5 \mu$ mdiameter with smallest particulates considered more dangerous as they penetrate deeper into the airways. In general cities have higher levels than sub-urban and rural areas. Levels are also affected by weather and high wind velocities decrease the levels.

Short term exposure causes irritation to eyes, nose throat and lungs leading to symptoms like coughing, wheezing and shortness of breath. Children with asthma may experience an acute attack on exposure.

Air filters in air conditioning and vacuum as well as wet mops help reduce indoor pollution.

Sulfates and Nitrates $\left(\mathrm{SO}_{2}\right.$ and $\left.\mathrm{NO}_{2}\right)$ : Emission of $\mathrm{SO}_{2}$ and $\mathrm{NO}_{2}$ into the atmosphere from factories and vehicular traffic causes deposition of acidic species of sulphates and nitrates. They are also byproducts of fossil fuel burning in home and contribute to particulates also as particles arising primarily from accretion of sulphates and nitrates are $2.5 \mu \mathrm{m}$ or less.

\section{Conclusion}

It is a matter of great concern that the quality of air that we breathe is getting poorer and has reached toxic levels. For a common person with limited resources, it is very difficult to make preventive steps as they are costly and beyond their reach. Substantial effort is required by the professionals, social organizations as well as governments to overcome these issues and that is at least what we to our children and future generations.

\section{References}

1. World Health Organization (WHO) Burden of disease from joint effects of household and ambient air pollution for 2012 . November 2016.

http://www.who.int/airpollution/data/cities/en/;accessed May 17, 2018.

2. World Health Organization (WHO) WHO ambient (outdoor) air quality database summary results, update 2018. Version April 6, 2018. Release date May 15. Accessed May 21, 2018. http://www.who.int/airpollution/data/cities/en

3. Dixon JK. Kids need clean air: air pollution and children's health. Fam. Community Health $2002 ; 24: 9-26$. 
4. World Health Organization (WHO): health environment in sustainable development five years after the earth summit. Geneva. 1997.

5. Landrigan PJ, Risk assessment for children and other sensitive population. Ann NYAcad Sci. 1999;895:1-9.

6. Etzel RA. The "fatal four" indoor air pollutant. Pediatr Ann 2000;29:344-50.

7. Reives D. Suffer the children. Chest. 2002;122:394-6.

8. Anderson ME, Bogdan GM. Environmrnts, Infdoor air quality and children. Pediatr Cl N Amer. 2007;54:295307.

9. Kattwinkel J, Hauck F, Keenan M et al. Task force on SIDS. The changing concept of SIDS: diagnostic coding shifts, controversies regarding the sleeping environment and new variables to consider in reducing risk. Pediatrics. 2005;116:1245-155.

10. Harold E Hoffman, Buka Irena, Phillips Scott. Medical Laboratory Investigation of children's Environments. Pediatr Cl N Amer. 2007;54:399-415.

11. Centers for disease control and prevention (CDC), 1991. Current trends in acute and chronic poisoning from residential exposures to elemental mercury-Michigan. 1989-1990. MMWR Morb Mort Wkly rep. 40:393-95.

12. Bush R, Portnoy J, Saxon A et al. The Medical Effects of Mold Exposure. J Allergy Clin Immunology 2006;117(2):326-33.

13. Canfield RI, Henderson CR, Cory-Slechta DA et al. Intellectual impairment in children with blood lead concentrations below 10 microgram per deciliter. $N$ Engl J Med 2003;348:1517-26. 